\title{
Mindfulness-based stress reduction for treating chronic headache: A systematic review and meta-analysis
}

Cephalalgia

2019, Vol. 39(4) 544-555

(C) International Headache Society 2018

Article reuse guidelines:

sagepub.com/journals-permissions DOI: $10.1177 / 03331024 \mid 8781795$

journals.sagepub.com/home/cep

(S)SAGE

\author{
Dennis Anheyer', Matthew J Leach ${ }^{2,3}$, Petra Klose', \\ Gustav Dobos' and Holger Cramer ${ }^{1,3}$
}

\begin{abstract}
Background: Mindfulness-based stress reduction/cognitive therapy are frequently used for pain-related conditions, but their effects on headache remain uncertain. This review aimed to assess the efficacy and safety of mindfulness-based stress reduction/cognitive therapy in reducing the symptoms of chronic headache.

Data sources and study selection: MEDLINE/PubMed, Scopus, CENTRAL, and PsychINFO were searched to I6 June 2017. Randomized controlled trials comparing mindfulness-based stress reduction/cognitive therapy with usual care or active comparators for migraine and/or tension-type headache, which assessed headache frequency, duration or intensity as a primary outcome, were eligible for inclusion. Risk of bias was assessed using the Cochrane Tool.

Results: Five randomized controlled trials (two on tension-type headache; one on migraine; two with mixed samples) with a total of 185 participants were included. Compared to usual care, mindfulness-based stress reduction/cognitive therapy did not improve headache frequency (three randomized controlled trials; standardized mean difference $=0.00 ; 95 \%$ confidence interval $=-0.33,0.32$ ) or headache duration (three randomized controlled trials; standardized mean difference $=-0.08$; $95 \%$ confidence interval $=-1.03,0.87)$. Similarly, no significant difference between groups was found for pain intensity (five randomized controlled trials; standardized mean difference $=-0.78$; 95\% confidence interval $=-1.72,0.16$ ).

Conclusions: Due to the low number, small scale and often high or unclear risk of bias of included randomized controlled trials, the results are imprecise; this may be consistent with either an important or negligible effect. Therefore, more rigorous trials with larger sample sizes are needed.
\end{abstract}

\section{Keywords}

Migraine, tension-type headache, complementary therapies

Date received: 17 January 2018; revised: 27 April 2018; accepted: II May 2018

\section{Background}

Chronic headache represents a group of disorders characterized by the presence of cephalic pain for at least 15 days per month, spanning a period of more than 3 months (1). Included within this collective are chronic migraine, chronic tension-type headache (TTH) and chronic cluster headache. The global prevalence of these disorders is high, with mean prevalence rates of all headache, TTH, migraine and chronic daily headache reported to be $47 \%, 38 \%, 10 \%$ and $3 \%$, respectively (2).

Chronic headache is not only a widespread condition, but one that carries a significant disease burden. Headache disorders are currently the sixth leading cause of disability across the globe (based on years lived with disability), and in 15 to 49-year-olds are the third leading cause of disability (3). Quality of life is also considerably reduced in individuals with a headache disorder, with chronic headache sufferers

\footnotetext{
'Department of Internal and Integrative Medicine, Kliniken Essen-Mitte, Faculty of Medicine, University of Duisburg-Essen, Essen, Germany

${ }^{2}$ Department of Rural Health, Division of Health Sciences, University of South Australia, Adelaide, South Australia, Australia

${ }^{3}$ Australian Research Centre in Complementary and Integrative Medicine (ARCCIM), Faculty of Health, University of Technology Sydney, Sydney, New South Wales, Australia
}

\section{Corresponding author:}

Dennis Anheyer, Department of Internal and Integrative Medicine, Kliniken Essen-Mitte, Faculty of Medicine, University of Duisburg-Essen, Am Deimelsberg 34a, 45276 Essen, Germany.

Email: d.anheyer@kliniken-essen-mitte.de 
reporting significantly lower quality of life than sufferers of episodic headache (4). The economic impact of this condition is noteworthy; in the US, migraine alone contributes to 86.5 million lost workdays annually, at an indirect annual cost of $\$ 9.3$ billion (5). The psychological, social, physiological and economic implications of headache are therefore substantial.

There are a number of modifiable and non-modifiable risk factors associated with the development of chronic headache, including stress, diet (e.g. caffeine overuse), sleep disturbance, obesity, psychiatric comorbidities and medication overuse (6). Notwithstanding, the management of this disorder is often symptomatically focused, with data from US epidemiological studies revealing that half of patients that present to an outpatient department with headache leave with a prescription for antimigraine medication (7). Interestingly, patients with headache generally do not view symptomatic management as a priority, with the cause of the headache seen to be of greater concern $(8,9)$. Not only does a symptomatic approach fail to address the origin of the condition, and to some extent a patient's needs, it also increases the risk of harm to patients. These harms include an increased risk of medication overuse headache, gastrointestinal bleeding (non-steroidal anti-inflammatory drugs), liver damage (acetaminophen), dependency (opioids), memory impairment, depression (topiramate), weight gain, and precipitation of cardiac arrhythmias and seizures (amitriptyline) (10,11).

In light of the abovementioned concerns regarding headache medication, there is a need for safer, effective treatment options for headache; in particular, the need for treatments that target the aetiology of headache. Focusing on just one of these factors - stress - there is increasing recognition that stress (particularly stressful life events) plays an integral role in the initiation, exacerbation and chronification of headache (12). Whilst the mechanisms of action have not been wellestablished, hypotheses suggest that stress contributes to nociceptor sensitization, and to the development of other risk factors of headache, such as sleep impairment, obesity and psychopathology (12). Thus, stress reduction would appear to be an important treatment strategy for chronic headache, and indeed, there is an emerging evidence base to support this view (13).

Non-pharmacological approaches that have been shown to effectively reduce stress (14), as well as chronic pain (15-17), are mindfulness-based stress reduction (MBSR) and mindfulness-based cognitive therapy (MBCT). These safe, highly standardized, effortless interventions, which are underpinned by Buddhist meditation traditions (16), have gained popularity in recent years, with lifetime prevalence of use in the
US, for any health condition, determined to be $2.5 \%$ (or 5.7 million US adults) (18). Notwithstanding the favorable safety profile and popularity of MBSR and $\mathrm{MBCT}$, and the emerging evidence-base supporting the effectiveness of these approaches for various conditions, the evidence of effectiveness of MBSR and MBCT for chronic headache is not clear, with no recent synthesis of the evidence knowingly published. In order to inform future policy, practice, research and education regarding the management of this condition, it is important to determine whether MBSR and MBCT are suitable treatment options for people suffering from chronic headache. This paper utilizes the process of systematic review and meta-analysis to address this knowledge gap. This review was designed to assess whether MBSR/MBCT is safe and effective in reducing the frequency, duration and/or intensity of headache in persons with chronic headache.

\section{Methods}

\section{Study design}

This systematic review and meta-analysis was conducted in accordance with the PRISMA statement (19) and the Cochrane handbook for systematic reviews of interventions (20). Prospective registration of the review protocol was not undertaken.

\section{Data sources and searches}

The following electronic databases were searched from their inception to 16 June 2017: MEDLINE/PubMed, Scopus, CENTRAL, and PsycINFO. EMBASE was not searched separately as it was included in Scopus. The search terms used for MEDLINE were as follows: (MBSR[Title/Abstract] OR MBCT[Title/Abstract] OR mindful ${ }^{*}$ [Title/Abstract] $O R$ mindfulness[Mesh] OR meditation*[Title/Abstract] OR meditation[Mesh]) AND (Tension-Type Headache[MeSH Terms] OR Tension-Type Headache[Title/Abstract] OR TTH [Title/Abstract] OR Migraine with Aura[MeSH Terms] OR Migraine without Aura[MeSH Terms] OR migraine* $^{*}$ Title/Abstract]). These search terms were adapted for the remaining databases as needed. Only articles published in the English or German language were eligible. The reference lists of identified original articles or reviews were also manually searched for relevant articles.

\section{Study selection}

Studies were eligible for inclusion in this review if they: (a) Were a randomised controlled trial, (b) included 
patients with tension-type headache and/or migraine, (c) compared MBSR or MBCT interventions to either a passive comparator (usual care) or an active comparator (e.g. health education or cognitive behavioral therapy); and (d) assessed headache frequency, duration and/or intensity as a primary outcome. Secondary outcomes of interest were mindfulness, safety and patient adherence. Studies were excluded if they: (a) Were observational studies, non-randomized trials or pseudorandomized trials, (b) tested interventions that differed clearly from the original MBSR/ MBCT programs (e.g. acceptance and commitment therapy or dialectic behavioral therapy), and/or (c) had not been published as full-text articles in peerreviewed scientific journals. The review was limited to RCTs as they represent the highest level of primary research evidence for clinical interventions. Further, RCTs are designed to control for both known and unknown confounders, and to establish a clear causal link between treatment and outcome (20).

The search was performed by DA. Abstracts identified during the literature search were screened independently by two review authors (DA, PK). Potentially eligible articles were also read in full-text by two authors (DA, PK), independently. Discrepancies between authors were rechecked and, if necessary, discussed with a third reviewer (HC) until consensus was achieved.

\section{Data extraction and quality assessment}

Data from included studies were independently extracted by two reviewers (DA, PK). Data extracted included: Study characteristics (e.g. trial design, randomization, blinding); patient population characteristics (e.g. sample size, age, diagnosis); characteristics of the intervention and comparison treatments (e.g. type, program length, frequency and duration); information about 'drop-outs'; selected outcome measures; results of short- and long-term follow-up; patient safety and adherence. Any discrepancies between reviewers were rechecked and discussed until consensus was reached.

\section{Risk of bias in individual studies}

The risk of bias of included studies was independently assessed by two authors (DA, PK), using the Cochrane Risk of Bias Tool. This tool assesses risk of bias as either low, unclear or high risk in the following domains: Selection, performance, detection, attrition, reporting and other biases (20). Discrepancies between reviewers were discussed until consensus was achieved and, if necessary, checked by a third reviewer (HC). The original trial authors were contacted for further details if necessary.

\section{Data synthesis and analysis}

Meta-analyses were conducted if at least two of the studies had sufficient statistical and clinical homogeneity to assess one of the aforementioned primary outcomes. Standardized mean differences (SMD), with $95 \%$ confidence intervals $(\mathrm{CI})$, were calculated as the difference in means between groups, divided by the pooled standard deviation. Where no standard deviations were cited, they were calculated from the standard errors, confidence intervals or t-values presented (20), or attempts were made to obtain this information from the original trial authors. Random effects meta-analysis estimates were obtained using $\mathrm{R}$ software Version 3.3.2 (R Foundation for Statistical Computing, Vienna, Austria. URL https://cran.r-project.org) and the 'metafor' package (written by Wolfgang Viechtbauer; http://www.metafor-project.org/). The Knapp-Hartung small-sample correction was used because it provided a more adequate account of uncertainty when pooling treatment effects from a small number of heterogeneous studies (21-24). For better comparability and interpretation of the results, the common DerSimonian-Laird estimator was additionally added to the forest plots.

With regard to headache frequency, duration and pain intensity, a negative SMD indicated beneficial effects for the MBSR/MBCT group when compared with the comparison group. For mindfulness, a positive SMD indicated beneficial effects for the mindfulness intervention.

Statistical heterogeneity between studies was explored using $\mathrm{I}^{2}$ and $\mathrm{Chi}^{2}$ statistics. An $\mathrm{I}^{2}>25 \%$, $\mathrm{I}^{2}>50 \%$ and $\mathrm{I}^{2}>75 \%$ were indicative of moderate, substantial and considerable heterogeneity, respectively (20). A $p$ value $\leq 0.10$ from the $\mathrm{Chi}^{2}$ test indicated significant statistical heterogeneity (20).

\section{Risk of bias across studies}

Publication bias was expected to be assessed via visual inspection of funnel plots. However, because of the small number of eligible trials (i.e. $<10$ trials), this was not possible. As a result, publication bias could not be assessed in this review (25). Similarly, sensitivity and subgroup analyses were not able to be performed.

\section{Results}

A total of 175 articles were identified from the literature search. After eliminating duplicates, 119 potentially relevant articles remained. Another 108 articles were eliminated during abstract screening as they did not meet the selection criteria (including 77 non-randomized controlled trials and 31 articles that were off topic). 


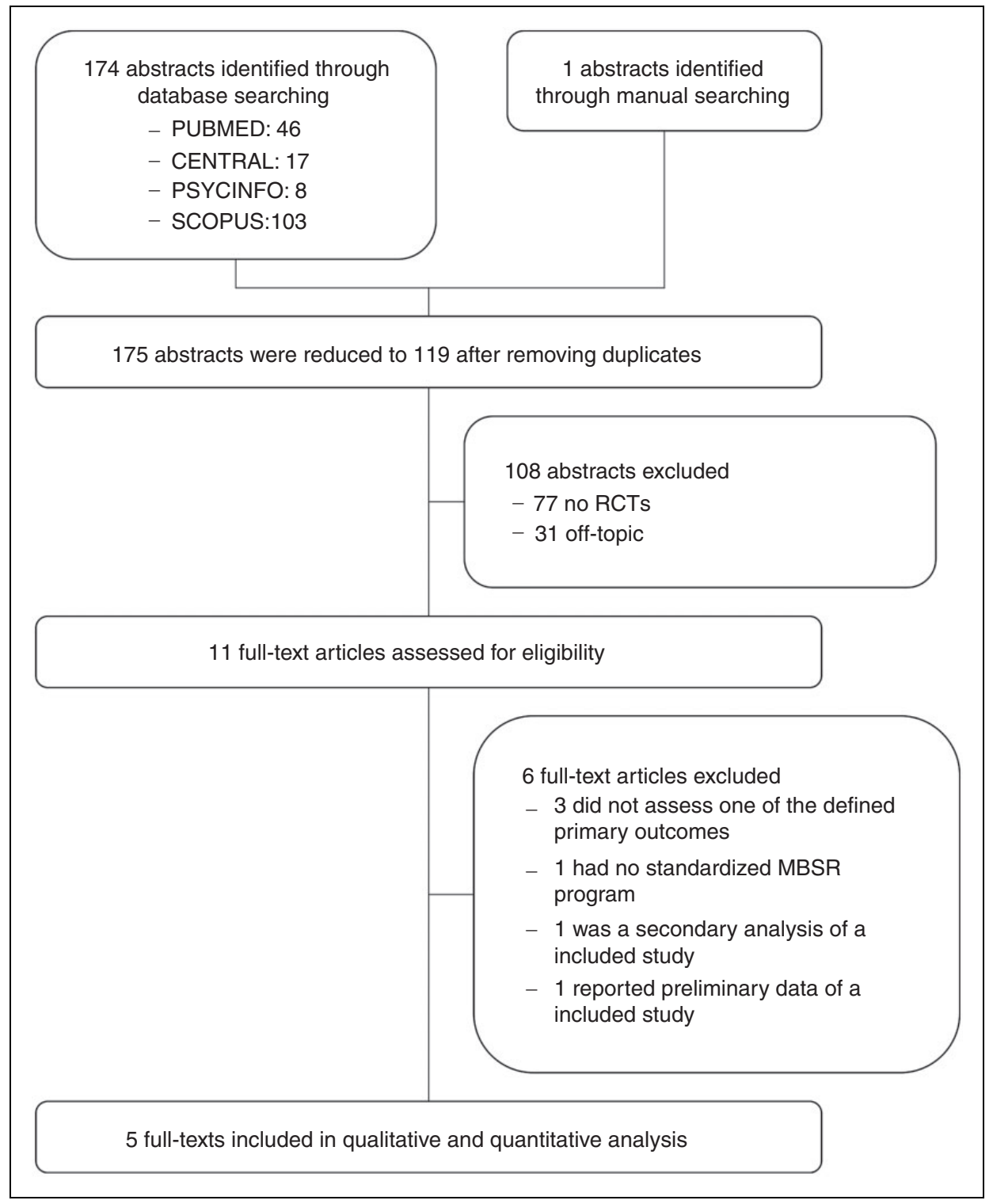

Figure I. Flow-chart of literature search.

The remaining 11 articles were then subjected to full-text screening. Six articles were excluded after full-text screening (Supplemental Table 1). Three articles did not assess the required outcomes (26-28), one study did not use a standardized MBSR/MBCT program (29), one article was a secondary analysis of an included trial (30), and one article only reported preliminary data (31). Five RCTs with a total of 185 patients were subsequently included in this review (Figure 1).

\section{Study characteristics}

Table 1 presents the study characteristics of the five RCTs included in this review. Two RCTs were conducted in Iran (32,33), two in the United States of America (34,35), and one in Australia (36).
Patients had been recruited from a university hospital (32), general hospital (33) and from the general population (34-36). Patients in all included RCTs were aged between 18 and 65 years, and had a diagnosis of chronic primary headache according to the International Classification of Headache Disorders (ICHD-II) criteria. Two studies included patients with tension-type headache and migraine $(32,35)$, two studies included patients diagnosed with tension-type headache only $(33,36)$, and one study included patients diagnosed with migraine only (34). Patients were diagnosed by neurologists $(32,33,34)$, psychiatrists $(32,33)$, psychologists (36) and general physicians (35).

All studies used headache diaries to assess frequency, duration and intensity of headaches. To assess mindfulness, two studies used the Mindfulness Attention 


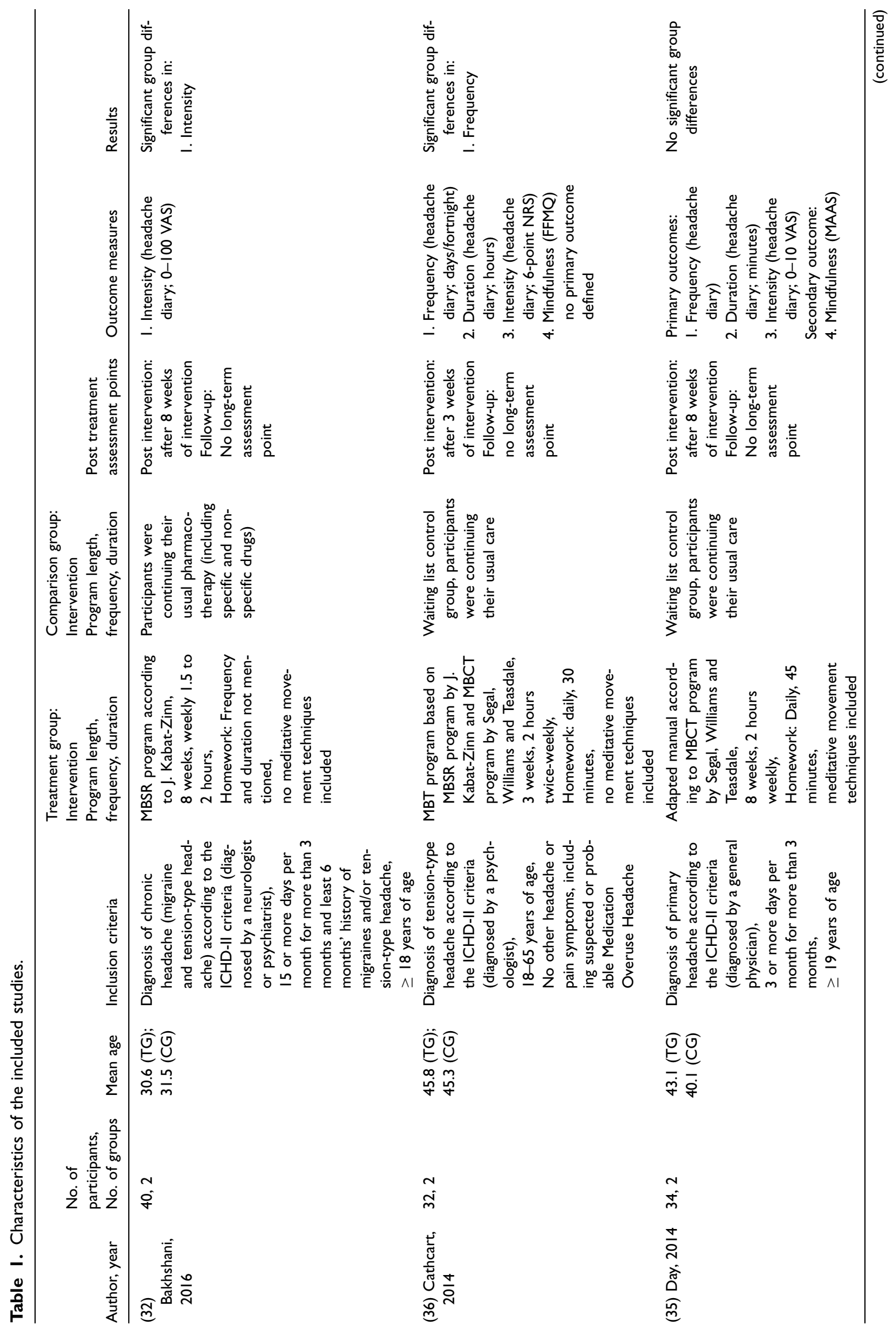




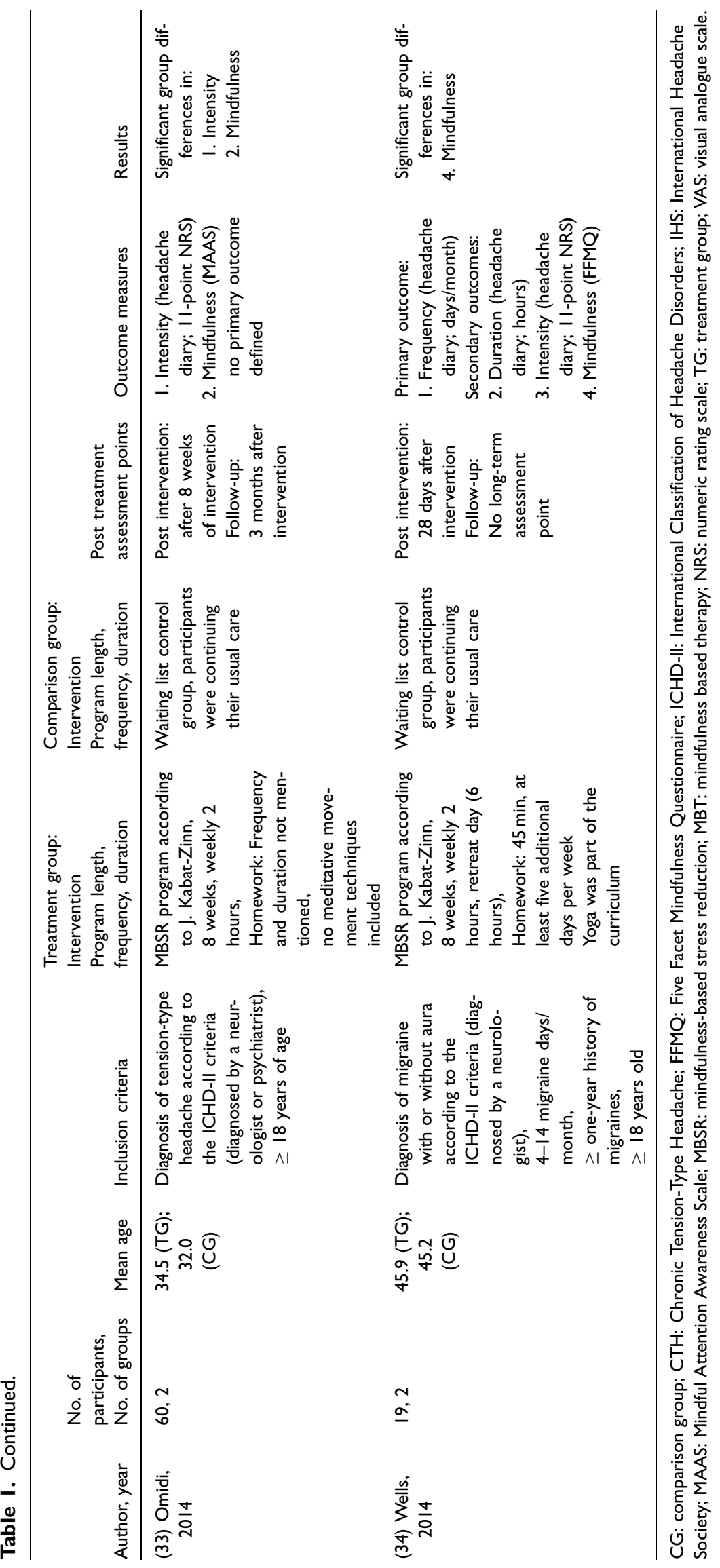


Awareness Scale (MAAS) $(33,35)$, and two used the Five Facet Mindfulness Questionnaire (FFMQ) $(34,36)$.

Three RCTs involved MBSR interventions that were adapted from the original MBSR program developed at the University of Massachusetts. One study used an adaption of the mindfulness-based cognitive therapy (MBCT) program developed by Segal, Williams and Teasdale (35), and one RCT used an adaption of the original MBSR program in combination with an adaption of the original MBCT program (36). Four trials modified the 8-week program by administering $90-120$ minute weekly sessions (instead of 150-minute weekly sessions) (32-35), while one trial administered 120-minute sessions twice weekly (36). All RCTs dedicated almost half of each session to mindful meditation (i.e. body scanning, sitting meditation, walking meditation), while the other half of the session was devoted to education and discussion. Only one RCT offered an optional 6-hour silent retreat (34). Daily 'homework' of 30-45 minutes meditation was recommended for at least five days a week in three of the included trials (34-36). The other two trials made no recommendations about frequency and length of 'homework'. Only two trials included yoga as a meditative movement technique in the offered program $(34,35)$.

All RCTs compared MBSR/MBCT plus usual care to usual care alone. No active comparator was used. Only one of the reviewed trials included a follow-up period (i.e. three months) after the intervention (33).

Only two of the included studies reported on funding $(34,35)$. Both studies were supported by grants of organizations that have no connections with the intervention. One study was supported by the Anthony Marchionne Foundation and the National Headache Foundation (35). The other study received grants from the American Headache Society and the Headache Research Fund of the John Graham Headache Center (34).

\section{Risk of bias}

The risk of bias for each study is shown in Table 2 . A detailed table with reasons for each judgement is provided as supplemental material (Supplemental Table 2). While only one of the reviewed RCTs reported adequate random sequence generation and allocation concealment (selection bias) (34), four studies did not clearly describe the randomization process $(32,33,35,36)$ and three studies did not report an adequate form of allocation concealment $(32,33,35)$. One study attempted to 'blind' its participants to treatment allocation by telling participants that there were two start times for the MBSR course, with randomization to either date (34). For three studies, the lack of information meant blinding of participants and personnel was unclear $(32,36,33)$, and for one study, risk of performance bias was judged as high (35). Only one study (36) reported 'blinding' of outcome assessments. The risk of attrition bias was considered high in one of the four RCTs (36) and the risk of reporting bias was adjudged high in two of the reviewed studies $(32,33)$. Other forms of bias were suspected in one of the included RCTs (35).

\section{Analyses of overall effects}

Effects on primary outcomes. Meta-analyses were performed to compare the effects of MBSR/MBCT and the comparison intervention on headache frequency, duration and pain intensity. The results of these metaanalyses are presented in Figure 2. Separate analyses by headache type are presented in Table 3.

Compared to usual care, MBSR/MBCT was not associated with a statistically significant improvement in headache frequency (three RCTs; $\mathrm{SMD}=0.00$; $95 \%$ CI -0.33 to 0.32 ) or headache duration (three RCTs; SMD $=-0.08 ; 95 \% \mathrm{CI}-1.03$ to 0.87 ).

Table 2. Risk of bias assessment.

\begin{tabular}{|c|c|c|c|c|c|c|c|}
\hline $\begin{array}{l}\text { Author, } \\
\text { year }\end{array}$ & $\begin{array}{l}\text { Random } \\
\text { sequence } \\
\text { generation } \\
\text { (selection bias) }\end{array}$ & $\begin{array}{l}\text { Allocation } \\
\text { concealment } \\
\text { (selection } \\
\text { bias) }\end{array}$ & $\begin{array}{l}\text { Blinding of } \\
\text { participants } \\
\text { and personnel } \\
\text { (performance } \\
\text { bias) }\end{array}$ & $\begin{array}{l}\text { Blinding of } \\
\text { outcome } \\
\text { assessment } \\
\text { (detection } \\
\text { bias) }\end{array}$ & $\begin{array}{l}\text { Incomplete } \\
\text { outcome data } \\
\text { (attrition bias) }\end{array}$ & $\begin{array}{l}\text { Selective } \\
\text { reporting } \\
\text { (reporting } \\
\text { bias) }\end{array}$ & $\begin{array}{l}\text { Other } \\
\text { bias }\end{array}$ \\
\hline [32] Bakhshani, 2016 & Unclear & Unclear & Unclear & Unclear & Unclear & High risk & Low risk \\
\hline [36] Cathcart, 2014 & Unclear & Low risk & Unclear & Low risk & High risk & Low risk & Low risk \\
\hline [35] Day, 2014 & High risk & High risk & High risk & High risk & Low risk & Low risk & High risk \\
\hline [33] Omidi, 2014 & Unclear & Unclear & Unclear & Unclear & Low risk & High risk & Low risk \\
\hline [34] Wells, 2014 & Low risk & Low risk & Low risk ${ }^{\mathrm{a}} /$ unclear $^{\mathrm{b}}$ & Unclear & Low risk & Low risk & Low risk \\
\hline
\end{tabular}

${ }^{a}$ Attempt to blind patients.

${ }^{\mathrm{b}}$ Therapists not blinded. 


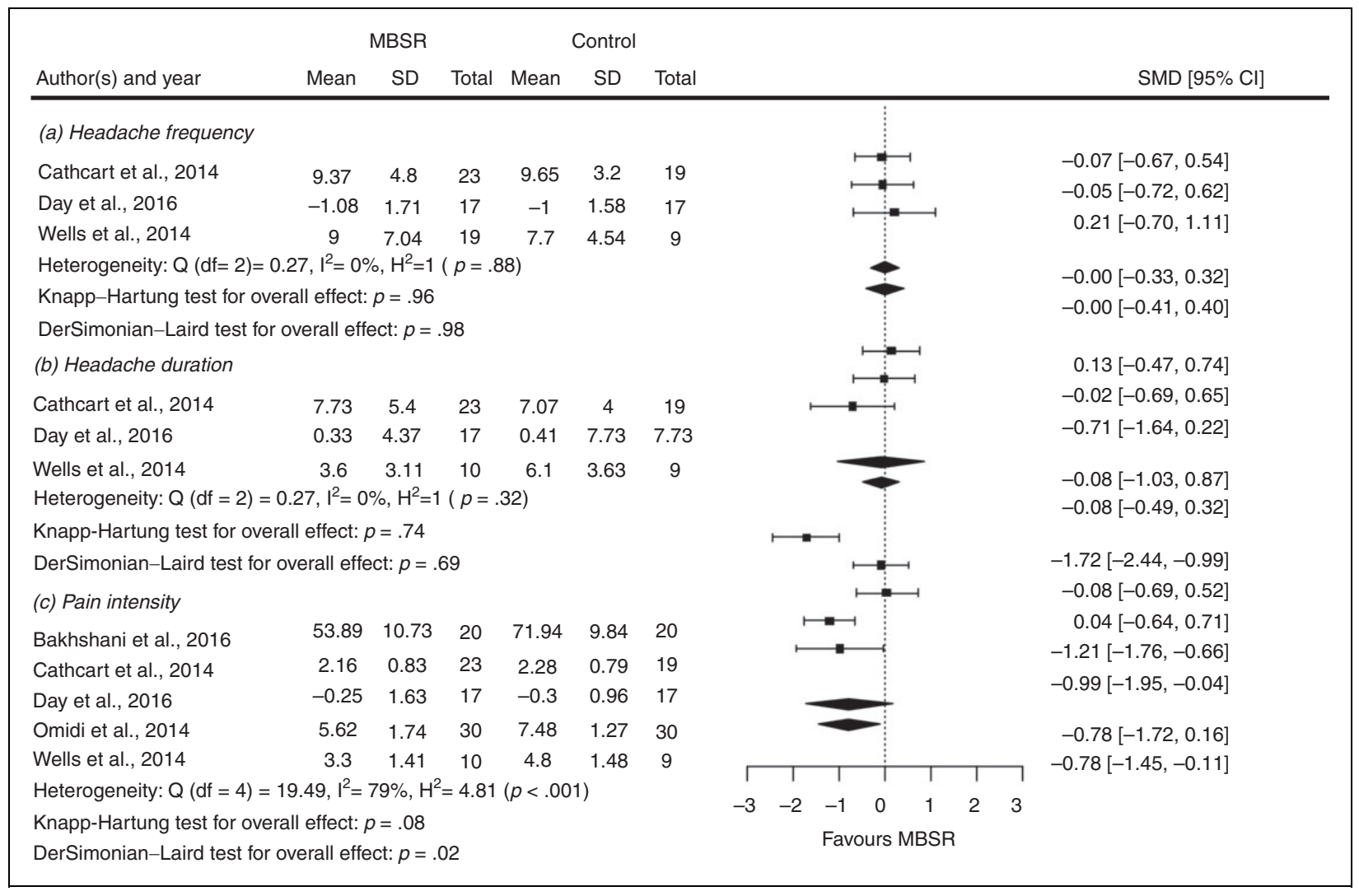

Figure 2. Meta-analysis of headache frequency, headache duration and pain intensity. (Knapp Hartung corrected). Cl: confidence interval; MBSR: mindfulness-based stress reduction; SD: standard deviation; SMD: standard mean difference.

Table 3. Analyses separated by headache type.

\begin{tabular}{|c|c|c|c|c|}
\hline Headache type & Headache frequency & Headache duration & Pain Intensity & Mindfulness \\
\hline \multicolumn{5}{|l|}{ Tension-type headache } \\
\hline Knapp-Hartung & $\begin{array}{l}\text { SMD }=-0.07 ; \\
\quad C I[-0.67 ; 0.54]\end{array}$ & $\begin{array}{l}\text { SMD }=0.13 ; \\
\quad C I[-0.47 ; 0.74]\end{array}$ & $\begin{array}{l}\mathrm{SMD}=-0.65 ; \\
\quad \mathrm{Cl}[-7.77 ; 6.47]\end{array}$ & $\begin{array}{l}\text { SMD }=0.12 ; \\
\quad C I[-2.14 ; 2.39]\end{array}$ \\
\hline DerSimonian-Laird & $\begin{array}{l}\text { SMD }=-0.07 ; \\
\quad C I[-0.67 ; 0.54]\end{array}$ & $\begin{array}{l}\text { SMD }=0.13 ; \\
\quad C I[-0.47 ; 0.74]\end{array}$ & $\begin{array}{l}\text { SMD }=-0.65 ; \\
\quad C l[-1.75 ; 0.45]\end{array}$ & $\begin{array}{l}\text { SMD }=0.12 ; \\
\quad C l[-0.27 ; 0.51]\end{array}$ \\
\hline Heterogeneity & $\begin{array}{l}\text { Only one RCT included } \\
\text { in this analysis. }\end{array}$ & $\begin{array}{l}\text { Only one RCT included } \\
\text { in this analysis. }\end{array}$ & $\begin{array}{l}\mathrm{I}^{2}=86 \% \\
\mathrm{H}^{2}=7.18(p=0.0 \mathrm{I}) \\
\text { two RCTs included. }\end{array}$ & $\begin{array}{l}\mathrm{I}^{2}=0 \% \\
\mathrm{H}^{2}=\mathrm{I}(p=0.37) \\
2 \text { RCTs included. }\end{array}$ \\
\hline \multicolumn{5}{|l|}{ Migraine } \\
\hline Knapp-Hartung & $\begin{array}{l}S M D=0.21 ; \\
\quad C I[-0.70 ; 1.1 I]\end{array}$ & $\begin{array}{l}\mathrm{SMD}=-0.71 ; \\
\quad \mathrm{Cl}[-1.64 ; 0.22]\end{array}$ & $\begin{array}{l}\text { SMD }=-0.99 ; \\
\quad C I[-1.95 ;-0.04]\end{array}$ & $\begin{array}{l}\text { SMD }=0.72 ; \\
\quad C I[-0.21 ; 1.65]\end{array}$ \\
\hline DerSimonian-Laird & $\begin{array}{l}\mathrm{SMD}=0.21 \\
\quad \mathrm{Cl}[-0.70 ; \mathrm{I} . \mathrm{II}]\end{array}$ & $\begin{array}{l}\mathrm{SMD}=-0.7 \mathrm{I} \\
\quad \mathrm{Cl}[-1.64 ; 0.22]\end{array}$ & $\begin{array}{l}\mathrm{SMD}=-0.99 \\
\quad \mathrm{Cl}[-1.95 ;-0.04]\end{array}$ & $\begin{array}{l}\mathrm{SMD}=0.72 ; \\
\quad \mathrm{Cl}[-0.21 ; 1.65]\end{array}$ \\
\hline Heterogeneity & $\begin{array}{l}\text { Only one RCT included } \\
\text { in this analysis. }\end{array}$ & $\begin{array}{l}\text { Only one RCT included } \\
\text { in this analysis. }\end{array}$ & $\begin{array}{l}\text { Only one RCT included } \\
\text { in this analysis. }\end{array}$ & $\begin{array}{l}\text { Only one RCT included } \\
\text { in this analysis. }\end{array}$ \\
\hline
\end{tabular}

Cl:95\% confidence interval; RCT: randomized controlled trial; SMD: standardized mean difference.

Notes: With regard to headache frequency, duration and pain intensity, a negative SMD indicates beneficial effects for MBSR/MBCT. For mindfulness, a positive SMD indicates beneficial effects for MBSR/MBCT. 


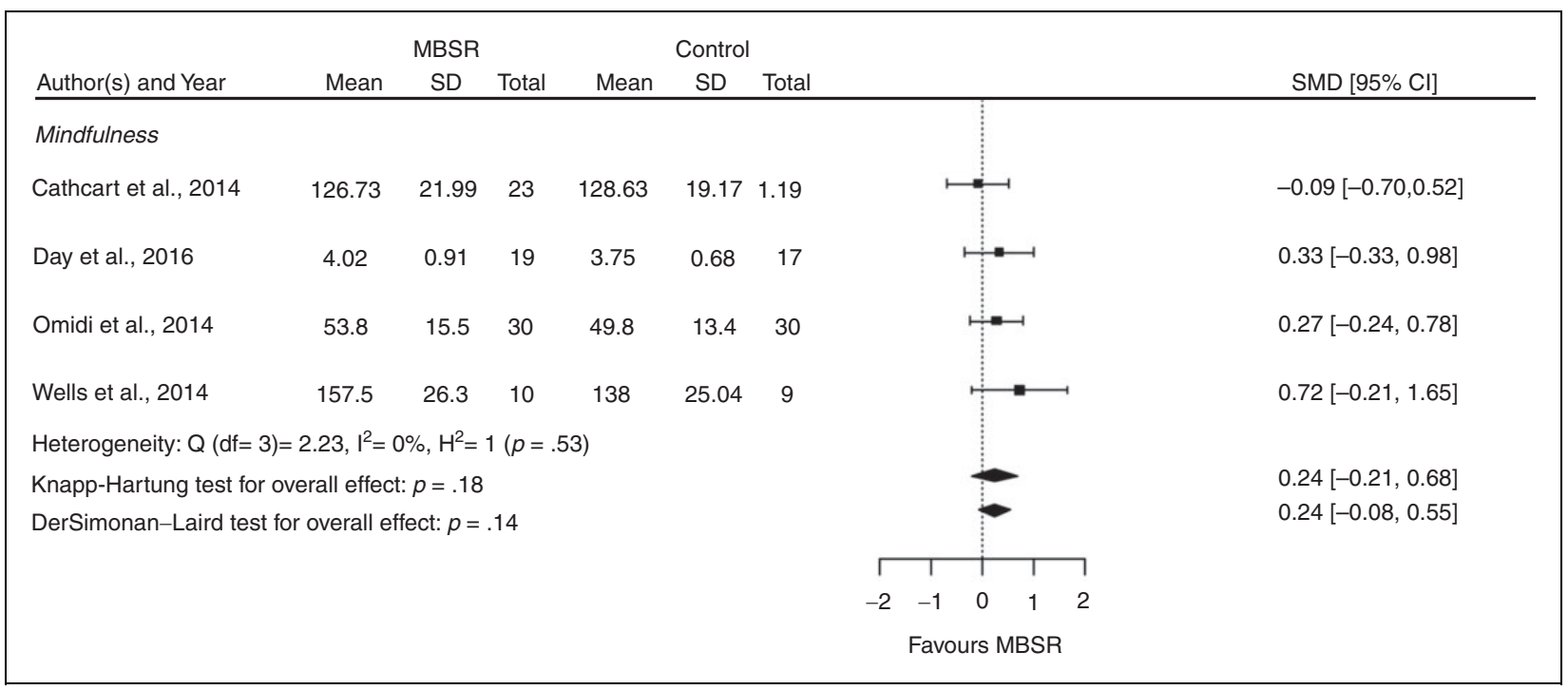

Figure 3. Meta-analysis of mindfulness. (Knapp Hartung corrected). Cl: confidence interval; MBSR: mindfulness-based stress reduction; SD: standard deviation; SMD: standard mean difference.

Separate analyses by individual headache type also showed no statistically significant differences in headache frequency and duration between MBSR/MBCT and control interventions. Similarly, no statistically significant differences between groups were found for pain intensity (five RCTs; SMD $=-0.78 ; 95 \% \mathrm{CI}-1.72$ to 0.16). However, using the DerSimonian-Laird estimator, the difference between groups in pain intensity was found to be statistically significant (SMD $=-0.78 ; 95 \%$ CI -1.45 to -0.11$)$. Moreover, a separate analysis by individual headache type showed a statistically significant improvement in pain intensity for migraine patients (one RCT; $\mathrm{SMD}=-0.99 ; 95 \% \mathrm{CI}-1.95$ to -0.04). None of the included RCTs reported on responder rates (i.e. $>50 \%$ reduction in headache days and duration).

Effects on secondary outcomes. For the outcome of mindfulness, the differences between MBSR/MBCT and usual care were not found to be statistically significant (four RCTs; SMD $=0.24 ; 95 \%$ CI -0.21 to 0.68 ) (Figure 3). A separate analysis by headache type also showed no statistically significant difference between intervention and control groups (Table 3).

Patient safety. Four RCTs did not report the occurrence (or absence) of adverse events $(32,33,35,36)$, and two studies did not provide any reasons for patient withdrawal (i.e. 'drop-outs') (33,36). One RCT reported that no adverse events had occurred and that no patients withdrew from the study as a result (34).

Patient adherence. Only two of the five RCTs reported on patient adherence to the MBSR/MBCT intervention
$(34,36)$. In one study, the mean number of MBSR classes attended was $5.1(\mathrm{SD}=.64)$ out of a possible six sessions (36). The other study reported an average attendance of $6.7(\mathrm{SD}=2.5)$ out of a possible nine sessions (including a retreat day) and an average daily meditation practice time of 34 minutes $(\mathrm{SD}=11)(34)$.

\section{Discussion}

\section{Summary of evidence}

The findings of this systematic review suggest that MBSR/MBCT is no more effective than usual care in reducing the frequency and duration of headache, or improving mindfulness, in patients with chronic headache. The results were largely imprecise, with the confidence intervals for headache frequency and headache duration containing medium or high effect sizes both in favor of MBSR/MBCT and in favor of usual care. Therefore, despite the low statistical heterogeneity between studies, no concrete conclusions can yet be drawn about the effectiveness of MBSR/MBCT for chronic headache.

Findings pertaining to pain intensity were also inconclusive. While the analysis using the Knapp-Hartung estimator found no statistically significant difference between groups, significant improvements in favor of MBSR/MBCT were observed using the DerSimonianLaird estimator. However, due to the low number of included studies, the relatively small overall population, high statistical heterogeneity and the associated uncertainty in the parameter of estimation, the results of the Knapp-Hartung corrected estimator can be considered more reliable. While a separate analysis by individual 
headache type showed a statistically significant improvement in pain intensity for migraine patients, the results of this analysis are based on only one RCT, and as such, should be interpreted with caution.

The available data on adherence and patient safety were also unsatisfactory. Although one study indicated that MBSR may be well tolerated, there was insufficient evidence to draw any firm conclusions about the safety of this therapy.

\section{Agreements with prior systematic reviews}

The results of this review partly concur with the findings of a recent meta-analysis of mindfulness-based stress reduction for chronic low back pain. The analysis found MBSR/MBCT to be superior to usual care in reducing pain intensity in the short term, but not in the long term (15). While the differences between MBSR/MBCT and usual care were found to be statistically significant, they were not clinically significant. The small sample sizes of the studies included in the current review may be one reason for the differences in the results. The analysis on low back pain, like the current review, also found MBSR/MBCT to be no more effective than usual care in improving participant mindfulness.

In contrast, other reviews have demonstrated preliminary evidence of effectiveness in support of MBSR for the management of long-term physical conditions (37), general pain disorders (38), and specific chronic pain disorders like fibromyalgia (17). Similarly, a large overview of meta-analyses on standardized mindfulnessbased interventions in healthcare (39) reported positive effects of MBSR/MBCT on the quality of life and physical functioning of patients with a wide range of chronic conditions. Since none of these reviews focused on headache pain in isolation, the results are only partially comparable.

\section{Limitations}

While this review was systematic and comprehensive, there were some limitations. Firstly, given the small number of RCTs included in this review, many of the meta-analyses included only two trials. As such, the results are likely to be imprecise (40), and consequently, the conclusions drawn from these meta-analyses should be considered preliminary. Secondly, the total number of participants in each included study was low, with only one study including at least 30 patients in each group. So, it is possible that the evidence of no effect for MBSR/MBCT could be indicative of a type II error in the included studies. This suggests that larger RCTs are needed to better understand the effectiveness of MBSR/MBCT for the treatment of chronic headache.
Thirdly, the risk of bias of included studies was largely unclear for most domains. Only one of the included studies was judged as having a low risk of bias in the most important domains (34). These potential biases should be taken into account when interpreting the findings of this review. Fourthly, only one of the included RCTs reported changes in medication intake during the study period (35). Therefore, the influence of pain medication on the study results cannot be precisely estimated.

\section{Implications for further research}

The limited number of trials examining the safety and effectiveness of MBSR/MBCT for chronic headache, and the high proportion of included studies with high/unclear risk of bias for most domains, highlights the need for more robust research in this area. For instance, none of the included RCTs used an active comparator. The use of an active comparator, such as cognitive behavioral therapy, would be helpful in understanding whether MBSR/MBCT is at least comparable to best practice care. Another consideration is that none of the included RCTs assessed the long-term safety or effectiveness of MBSR/MBCT. It could be assumed that the integration of MBSR/MBCT into everyday life may take time for some people, and as such, delayed effects should be expected. With regard to the study population, none of the included studies investigated the effectiveness of MBSR in specific age groups (i.e. children and adolescents; elderly). It is possible that different age groups understand mindfulness in distinct ways and utilize MBSR/MBCT differently in their everyday life; this could in turn lead to variable effects (e.g. delayed response or non-response to the intervention) for specific age groups.

Surprisingly, the meta-analysis found no significant difference in mindfulness between patients assigned to MBSR/MBCT or usual care. It is possible that the reliability of the outcomes measuring mindfulness were a contributing factor; it is also possible that mindfulness does not constitute a decisive factor in the improvement of chronic headache. Further research is needed to understand the potential mechanisms by which MBSR/MBCT may improve chronic headache to ensure these elements are adequately captured in future MBSR/MBCT training and research.

\section{Conclusions}

Despite the aforementioned limitations, this systematic review found no evidence that MBSR/MBCT is effective in improving the frequency, duration or intensity of headache in patients suffering from chronic headaches. Due to the small number of RCTs included in this 
review, the low total number of participants in each included study and the largely high/unclear risk of bias of included trials, the results of the meta-analyses are imprecise. That is, the wide confidence intervals in the analyses could be consistent with either an important or negligible effect. In light of these findings, the use of MBSR/MBCT interventions for the treatment of migraine and/or tension-type headache cannot yet be recommended. More rigorous trials with larger sample sizes and longer durations are needed before a definitive conclusion about the value of MBSR/MBCT in chronic headaches can be reached.

\section{Key findings}

- The use of MBSR/MBCT interventions cannot yet be recommended.

- More rigorous trials with larger sample sizes and longer durations are clearly needed.

\section{Author contributions}

DA was responsible for the conception/design of this review, carried out the literature search, performed the data extraction and analysis, assessed the risk of bias and drafted the resulting manuscript. ML and HC participated in the conception/design of the review, critically revised and drafted the resulting manuscript. PK performed the data extraction, assessed the risk of bias, and critically revised the manuscript. GD participated in the conception/design of the review and critically revised the manuscript. All authors approved the final manuscript prior to submission.

\section{Declaration of conflicting interests}

The authors declared no potential conflicts of interest with respect to the research, authorship, and/or publication of this article.

\section{Funding}

The authors disclosed receipt of the following financial support for the research, authorship, and/or publication of this article: This article was supported by a grant from the Karl and Veronica-Carstens Foundation. The funding source had no influence on the design or conduct of the review; the collection, management, analysis, or interpretation of the data; or in the preparation, review, or approval of the manuscript. The authors have no financial relationships, related to this article, to disclose.

\section{References}

1. The International Classification of Headache Disorders, 3rd edition (beta version). Cephalalgia 2013; 33: 629-808.

2. Stovner L, Hagen K, Jensen R, et al. The global burden of headache: A documentation of headache prevalence and disability worldwide. Cephalalgia 2007; 27: 193-210.

3. Steiner TJ, Stovner LJ and Vos T. GBD 2015: Migraine is the third cause of disability in under 50s. J Headache Pain 2016; 17: 104.

4. Ayzenberg I, Katsarava Z, Sborowski A, et al. Headacheattributed burden and its impact on productivity and quality of life in Russia: Structured healthcare for headache is urgently needed. Eur J Neurol 2014; 21: 758-765.

5. Kessler RC, Shahly V, Stang PE, et al. The associations of migraines and other headaches with work performance: Results from the National Comorbidity Survey Replication (NCS-R). Cephalalgia 2010; 30: 722-734.

6. Cho SJ and Chu MK. Risk factors of chronic daily headache or chronic migraine. Curr Pain Headache Rep 2015; 19: 465.

7. Smitherman TA, Burch R, Sheikh H, et al. The prevalence, impact, and treatment of migraine and severe headaches in the United States: A review of statistics from national surveillance studies. Headache 2013; 53 : 427-436.

8. Davies PTG, Glynn CJ and Kadry MA. Expectations of patients attending a combined headache clinic. $J$ Headache Pain 2003; 4: 79-82.

9. Thomas E, Boardman HF, Ogden H, et al. Advice and care for headaches: Who seeks it, who gives it? Cephalalgia 2004; 24: 740-752.

10. Couch JR. Update on chronic daily headache. Curr Treat Options Neurol 2011; 13: 41-55.

11. Loder E, Weizenbaum E, Frishberg B, et al. Choosing wisely in headache medicine: The American Headache Society's list of five things physicians and patients should question. Headache 2013; 53: 1651-1659.

12. Houle T and Nash JM. Stress and headache chronification. Headache 2008; 48: 40-44.

13. Penzien DB, Irby MB, Smitherman TA, et al. Wellestablished and empirically supported behavioral treatments for migraine. Curr Pain Headache Rep 2015; 19: 34 .

14. Khoury B, Sharma M, Rush SE, et al. Mindfulness-based stress reduction for healthy individuals: A meta-analysis. J Psychosom Res 2015; 78: 519-528.

15. Anheyer D, Haller H, Barth J, et al. Mindfulness-based stress reduction for treating low back pain: A systematic review and meta-analysis. Ann Intern Med 2017; 166: 799-807.

16. Greeson $\mathrm{J}$ and Eisenlohr-Moul T. Mindfulness-based stress reduction for chronic pain. In: Baer RA (ed.) Mindfulness-based treatment approaches: Clinician's guide to evidence base and applications, 2nd edn. Amsterdam: Elsevier, 2014, pp.269-292.

17. Lauche R, Cramer H, Dobos G, et al. A systematic review and meta-analysis of mindfulness-based stress reduction for the fibromyalgia syndrome. J Psychosom Res 2013; 75: 500-510. 
18. Cramer H, Hall H, Leach M, et al. Prevalence, patterns, and predictors of meditation use among US adults: A nationally representative survey. Sci Rep 2016; 6: 36760.

19. Moher D, Liberati A, Tetzlaff J, et al. Preferred reporting items for systematic reviews and meta-analyses: The PRISMA statement. Ann Intern Med 2009; 151: 264-269, W64.

20. Higgins JPT and Green S. Cochrane Handbook for systematic reviews of interventions, Version 5.1.0. The Cochrane Collaboration, 2011.

21. Cornell JE, Mulrow CD, Localio R, et al. Randomeffects meta-analysis of inconsistent effects: A time for change. Ann Intern Med 2014; 160: 267-270.

22. Knapp $G$ and Hartung J. Improved tests for a random effects meta-regression with a single covariate. Stat Med 2003; 22: 2693-2710.

23. IntHout $\mathbf{J}$, Ioannidis JP and Borm GF. The HartungKnapp-Sidik-Jonkman method for random effects meta-analysis is straightforward and considerably outperforms the standard DerSimonian-Laird method. BMC Med Res Methodol 2014; 14: 25.

24. Rover C, Knapp G and Friede T. Hartung-Knapp-SidikJonkman approach and its modification for randomeffects meta-analysis with few studies. BMC Med Res Methodol 2015; 15: 99.

25. Egger M, Davey Smith G, Schneider M, et al. Bias in meta-analysis detected by a simple, graphical test. $B M J$ 1997; 315: 629-634.

26. Cathcart S, Barone V, Immink M, et al. Mindfulness training does not reduce generalized hyperalgesia in chronic tension-type headache. J Pain Manage 2013; 6: 217-221.

27. Cathcart S, Vedova CD, Immink M, et al. Mindfulness training does not reduce inflammatory cytokine levels in chronic tension-type headache. J Pain Manage 2013; 6: 235-238.

28. Feuille M and Pargament K. Pain, mindfulness, and spirituality: A randomized controlled trial comparing effects of mindfulness and relaxation on pain-related outcomes in migraineurs. J Health Psychol 2015; 20: 1090-1106.

29. Wachholtz AB, Malone CD and Pargament KI. Effect of different meditation types on migraine headache medication use. Behav Med 2017; 43: 1-8.
30. Omidi A and Zargar F. Effects of mindfulness-based stress reduction on perceived stress and psychological health in patients with tension headache. J Res Med Sci 2015; 20: 1058-1063.

31. Andrasik F, Grazzi L, D'Amico D, et al. Mindfulness and headache: A "new" old treatment, with new findings. Cephalalgia 2016; 36: 1192-1205.

32. Bakhshani NM, Amirani A, Amirifard H, et al. The effectiveness of mindfulness-based stress reduction on perceived pain intensity and quality of life in patients with chronic headache. Glob J Health Sci 2015; 8: 142-151.

33. Omidi A and Zargar F. Effect of mindfulness-based stress reduction on pain severity and mindful awareness in patients with tension headache: A randomized controlled clinical trial. Nurs Midwifery Stud 2014; 3: e21136.

34. Wells RE, Burch R, Paulsen R, et al. Meditation for migraines: A pilot randomized controlled trial. Cephalalgia 2013; 33: 96-97.

35. Day MA, Thorn BE, Ward LC, et al. Mindfulness-based cognitive therapy for the treatment of headache pain: A pilot study. Clin J Pain 2014; 30: 152-161.

36. Cathcart S, Galatis N, Immink M, et al. Brief mindfulness-based therapy for chronic tension-type headache: A randomized controlled pilot study. Behav Cogn Psychother 2014; 42: 1-15.

37. Crowe M, Jordan J, Burrell B, et al. Mindfulness-based stress reduction for long-term physical conditions: A systematic review. Aust N Z J Psychiatry 2016; 50: 21-32.

38. Bawa FL, Mercer SW, Atherton RJ, et al. Does mindfulness improve outcomes in patients with chronic pain? Systematic review and meta-analysis. $\mathrm{Br} J$ Gen Pract 2015; 65: e387-e400.

39. Gotink RA, Chu P, Busschbach JJ, et al. Standardised mindfulness-based interventions in healthcare: An overview of systematic reviews and meta-analyses of RCTs. PLoS One 2015; 10: e0124344.

40. InyHout J, Ioannidis JPA, Rovers MM, et al. Plea for routinely presenting prediction intervals in meta-analysis. BMJ Open 2016; 6: e010247. 
DuEPublico

Duisburg-Essen Publications online
UNIVERSITÄT
DEUISSB R G G

offen im Denken

$\mathbf{U b} \mid \begin{aligned} & \text { universitäts } \\ & \text { bibliothek }\end{aligned}$

This text is made available via DuEPublico, the institutional repository of the University of Duisburg-Essen. This version may eventually differ from another version distributed by a commercial publisher.

DOI: $\quad 10.1177 / 0333102418781795$

URN: urn:nbn:de:hbz:464-20210511-161051-9

This publication is with permission of the rights owner freely accessible due to an Alliance licence and a national licence (funded by the DFG, German Research Foundation) respectively.

(C) International Headache Society 2018. All rights reserved. 